\title{
Edad y crecimiento de la mojarra Oreochromis aureus (Pisces: Cichlidae) en la Presa Sanalona, Sinaloa, México
}

\author{
Rigoberto Beltrán-Álvarez ${ }^{1}$, Jesús Sánchez-Palacios ${ }^{1}$, Guadalupe L. Valdez ${ }^{1}$ \\ \& Armando A. Ortega-Salas ${ }^{2}$ \\ 1. Laboratorio de Limnología y Pesquerías de Agua Dulce, FACIMAR, UAS. Paseo Claussen S/N, Colonia los Pinos, \\ Mazatlán, Sinaloa, México; rigober80@hotmail.com \\ 2. Instituto de Ciencias del Mar y Limnología, UNAM
}

Recibido 02-III-2009. Corregido 10-VII-2009. Aceptado 11-VIII-2009.

\begin{abstract}
Age and growth of Oreochromis aureus (Pisces: Cichlidae) in Sanalona Reservoir, Sinaloa, Mexico. Oreochromis aureus is an important fishing resource in this reservoir but little biological information is available for management purposes. To study its age and growth in the site, we obtained samples using gill nets of six mesh sizes, and collecting fish from commercial catches, on a bimonthly basis, from November 2006 to September 2007. Total length $\left(\mathrm{L}_{t}\right)$, total weight $\left(\mathrm{P}_{t}\right)$, sex and gonadosomatic maturity were analyzed. Age determination was done by readings of scale growth marks, and its validation was made by the marginal and gonadosomatic index. A total of 384 fish were collected. Length and weight varied from 125 to $345 \mathrm{~mm}$ and from 40 to $671 \mathrm{~g}$, respectively. Growth in length and weight are described by the following von Bertalanffy equations: $\mathrm{L}_{\mathrm{t}}=416\left[1-\mathrm{e}^{-0.3682(\mathrm{t}+0.4975)}\right]$ and $\mathrm{P}_{\mathrm{t}}=1323\left[1-\mathrm{e}^{-0.3682(\mathrm{t}+0.4975)}\right]^{3.0652}$. Two growth marks per year were registered, these were associated to metabolic and environmental factors like reproduction and temperature. Rev. Biol. Trop. 58 (1): 325-338. Epub 2010 March 01.
\end{abstract}

Key words: age, growth, Oreochromis aureus, reservoir, Mexico.

En los últimos años la creciente demanda nutricional que ha presentado nuestro país ha propiciado que la pesca en aguas continentales tenga mayor importancia, no sólo por la obtención de alimento de alto contenido proteico, sino además por la creación de fuentes de empleo. La introducción de especies exóticas como la tilapia (Oreochromis spp.) en los embalses ha mejorado los estándares de vida de las comunidades rurales (Contreras-MacBeath et al. 1998). La mayoría de las Presas en Sinaloa, además de apoyar el desarrollo agropecuario, han permitido el establecimiento de importantes pesquerías basadas en la explotación y comercialización de especies como Oreochromis aureus, Ictalurus punctatus y Micropterus salmoides, y dan sustento a un importante número de familias, todas ellas habitantes de la zona serrana (Beltrán 2003).
Los cíclidos se encuentran predominantemente en climas tropicales, donde las estaciones del año no son muy marcadas y el crecimiento de las especies tiende a ser continuo, esto implica cierta desventaja en la aplicación de los métodos de análisis de frecuencia por tallas para la determinación de edad, en tanto que las estructuras óseas pueden resultar muy útiles si se define claramente su periodicidad debido a factores ambientales o endógenos, que no siempre es anual como ocurre en especies de lugares templados (Weatherley \& Gill 1987, Morales-Nin 1989).

Estudios sobre edad y crecimiento son necesarios para realizar un adecuado manejo y explotación de los recursos pesqueros, ya que se requieren para describir y cuantificar la estimación de la mortalidad, tamaño de la población, reclutamiento, selectividad de los artes de 
pesca, edad de primera madurez, rendimiento máximo sostenible entre otras variables poblacionales relacionadas con su explotación, así como la tasa de crecimiento individual de los peces influye en la sobrevivencia, maduración sexual y la fecundidad (Jones 2002).

La historia del crecimiento individual de los peces se encuentra registrada en las estructuras duras como otolitos, escamas, vértebras, huesos y radios. De aquí que la edad puede estimarse en muchas especie ícticas a partir de las discontinuidades que ocurren en las estructuras esqueléticas que pueden deberse a cambios fisiológicos o por factores ambientales como temperatura, fotoperíodo, disponibilidad de alimento, entre otros (Morales-Nin 2000).

La determinación de la edad, a partir de la formación de marcas de crecimiento en las partes duras de los peces se relaciona directamente con la reducción del crecimiento durante períodos particulares (Carlander 1974). En este sentido, Pannella (1974) menciona que en las estructuras duras de peces tropicales ocurren periódicamente bandas de crecimiento que, una vez establecida la periodicidad de su formación, pueden ser utilizadas para la determinación de la edad.

De los cíclidos traídos a México destacan Oreochromis spp. y $O$. niloticus ya que forman parte de importantes pesquerías. Actualmente existen algunos estudios sobre su edad y crecimiento para otras zonas. Jiménez-Badillo (2006) describe el crecimiento de $O$. aureus en la presa El Infiernillo, México utilizando las marcas en escamas, opérculos y análisis de frecuencias de tallas. Por su parte, Gómez-Márquez et al. (2008) realizan un estudio similar para $O$. niloticus en el lago Cuatetelco, México, utilizando las marcas en el hueso opercular.

Ante la falta de información sobre aspectos biológicos y pesqueros de la tilapia $O$. aureus en la Presa Sanalona, el objetivo de la presente investigación fue determinar la edad y el crecimiento de esta especie.

\section{MATERIALES Y MÉTODOS}

La Presa Sanalona está localizada en el Estado de Sinaloa, Mexico (2449' 3 " N y $\left.107^{\circ} 10^{\prime} \mathrm{W}\right)$. Los muestreos para la colecta de los organismos se realizaron bimensualmente de noviembre de 2006 a septiembre del 2007 en distintos puntos del embalse. Con la finalidad de garantizar la representatividad de las muestras tanto en tallas, edades y sexos, así como disminuir el efecto de la selectividad del arte de pesca, la captura de los organismos se realizó mediante el empleo de redes agalleras de 2.0, 2.5, 3.0, 3.5, 4.0 y 4.5 pulgadas de luz de malla, las cuales se colocaron al atardecer y se recogieron al amanecer del día siguiente. Adicionalmente, se obtuvieron peces de la captura comercial. Una vez colectados, los organismos se separados y contados según la luz de malla de la red.

Se registraron los datos biométricos como la longitud total, longitud patrón y altura del cuerpo utilizando un ictiómetro convencional con divisiones de $1 \mathrm{~mm}$. El peso total se registró con una balanza digital (5 000g de capacidad y $1 \mathrm{~g}$ de precisión), y el peso de la gónada, con una balanza digital de $300 \mathrm{~g}$ de capacidad y precisión de $0.01 \mathrm{~g}$. El sexo se registró mediante la observación directa de las gónadas y su grado de madurez, utilizando la escala de maduración propuesta por Morales (1991), la cual describe cinco estadios de madurez de las gónadas de los peces: el estadio I corresponde a organismos inmaduros, el IV máxima madurez y el $\mathrm{V}$ organismos ya desovados. Para establecer si existía diferencia en las tallas alcanzadas entre sexos se utilizó la prueba KolmogorovSmirnof. La relación longitud total-peso total se ajustó a un modelo tipo potencial.

La edad se determinó mediante el conteo de marcas de crecimiento en las escamas obtenidas debajo las aletas pectorales y sobre la línea lateral de ambos costados, etiquetadas en bolsas de plástico con el número del pez. 
En el laboratorio, fueron lavadas con solución jabonosa, y una vez limpias, se seleccionaron las seis mejores y se colocaron entre dos portaobjetos sujetados con cinta (Tesh 1968, Ruiz-Dura et al. 1970). Las escamas se leyeron con un proyector de escamas marca Bausch \& Lomb mediante luz transmitida con objetivo de $3.2 \mathrm{X}$.

Para establecer la proporcionalidad que guardan las escamas con la longitud del pez, se relacionó el radio total $\left(\mathrm{R}_{\mathrm{t}}\right)$ contra la longitud total del pez $\left(\mathrm{L}_{\mathrm{t}}\right)$. La determinación de la longitud del pez a cada marca de crecimiento se realizó aplicando el método de retrocálculo mediante la fórmula de proporción directa de Lea (1920) modificada por Fraser (1916) y Lee (1920). La temporalidad de la formación de las marcas de crecimiento en las escamas se reconocieron analizando gráficamente los incrementos marginales (IM) promedio mensual por grupos de edad, el cual se define como la distancia de la última marca al borde de la escama (Bagenal \& Tesch 1978, Jearld 1983).

La formación de las marcas de crecimiento en las escamas se relacionó con factores endógenos como los periodos de actividad reproductiva identificados a través del seguimiento del índice gonadosomatico (IGS) según Rossenblum et al. (1987). A partir de la longitud promedio calculada a cada marca de crecimiento se construyó la clave edad-longitud. La obtención de los parámetros se realizó mediante el método de mínimos cuadrados lineales (Ford-Walford). Para definir el crecimiento en longitud y en peso se utilizaron las ecuaciones del modelo de von Bertalanffy (1938).

Para evaluar la confiabilidad de los parámetros de crecimiento de la ecuación de von Bertalanffy estimados en el presente estudio con respecto a investigaciones realizados en diferentes cuerpos de agua del mundo, se aplicó la "prueba de phi ( $\Phi$ ') prima" de Pauly (1983) y Pauly \& Munro (1984), utilizando los parámetros que definen la curva de crecimiento como son " $L \infty$ " y " $k$ ". Los valores de $\Phi$ se obtuvieron mediante la expresión matemática: $\Phi=\log (k)+2 * \log (L \infty)$.

\section{RESULTADOS}

Estructura de la población por talla y peso: El análisis de 384 especimenes de $O$. aureus mostró tallas y pesos que variaron de 125 a $345 \mathrm{~mm}$ y 39 a $671 \mathrm{~g}$, respectivamente. El examen por sexos reveló que las hembras presentaron tallas desde los 125 a $307 \mathrm{~mm}$ y pesos de 40 a $539 \mathrm{~g}$, en tanto que los machos la variación fue de 131 a $315 \mathrm{~mm}$ y de 39 a $530 \mathrm{~g}$, respectivamente (Fig. 1). Los machos resultaron ser estadísticamente más grandes que las hembras (prueba U de Mann-Whitney, $U=8998.5$; $P<0.001$ ), por lo que las ecuaciones de crecimiento se calcularon para cada sexo.

Relación longitud total-peso total: El modelo fue de tipo potencial el cual se expresó como: $\mathrm{P}_{\mathrm{t}}=0.0000124\left(\mathrm{~L}_{\mathrm{t}}\right)^{3.0652}$ con un grado de asociación entre estas dos variables del 99.04\% $(r=0.9904)$ significativa para una $p<0.001$ (Fig. $2)$. La prueba $t$-student indica que el valor del exponente $(b=3.0652)$, fue significativamente diferente de 3 ( $t=2.9858, p<0.001)$, por lo que el crecimiento de la especie en este embalse se consideró como alométrico positivo.

Relación radio de la escama - longitud total del pez: La relación entre estas variables quedó como sigue: $\mathrm{L}_{\mathrm{t}}=49.09\left(\mathrm{R}_{\mathrm{t}}\right)+9.277$, $r=0.9649$ (Fig. 3). Lo que permite establecer una relación directa entre ambas variables.

\section{Retrocálculo de longitudes pretéritas:} Los valores estimados de longitud total por cada marca de crecimiento y los valores promedios de longitud total para cada grupo de edad, permitieron establecer que 30 organismos registraron seis marcas, 114 cinco, 71 cuatro, 37 tres, 9 dos y 4 una.

\section{Validación de las marcas de crecimiento:} La validación de las marcas de crecimiento en las estructuras duras es uno de los aspectos importantes a establecer en la determinación de la edad, ya que permite asignar la temporalidad 

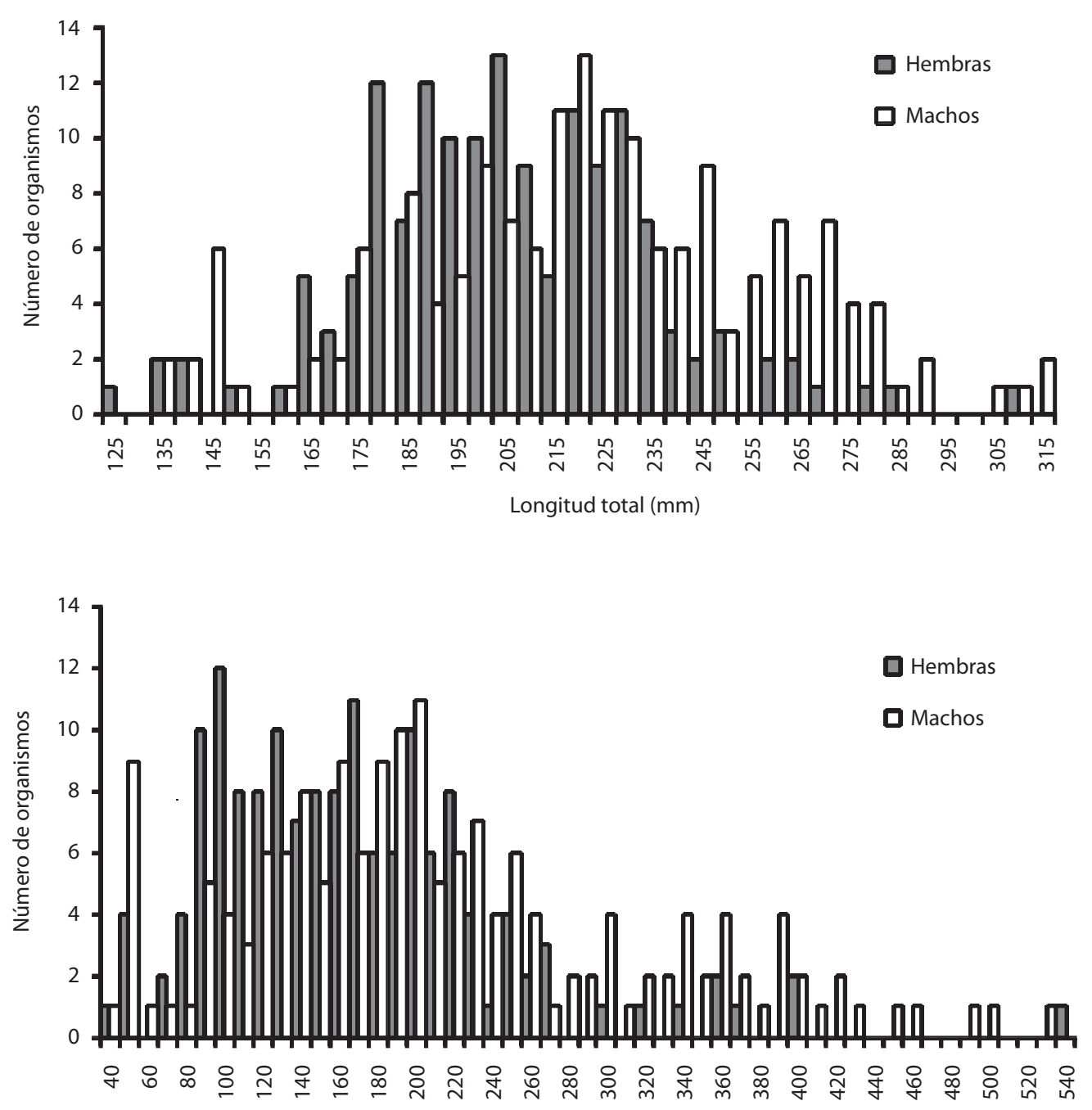

Peso total (g)

Fig. 1. Distribución de frecuencias de longitud y peso total.

Fig. 1. Length and total weight: frequency distribution.

de su formación y en consecuencia la determinación de la edad. Para este estudio se consideraron el índice marginal (IM) y el índice gonadosomático (IG). Al analizar la evolución del IM promedio mensual en el tiempo (Fig. 4), se observó que los menores valores se presentaron alrededor de enero, lo cual indica que una de las marcas de crecimiento se presenta precisamente en este periodo de tiempo, probablemente por la temperatura de la capa superficial a seis metros, que se registra en promedio a $20^{\circ} \mathrm{C}$. En los meses siguientes, se observa un incremento en los valores del IM los cuales se mantienen de marzo a mayo. Igualmente se presenta una disminución de los valores alrededor de julio, revelando otro periodo de formación de marca de crecimiento. En general el comportamiento del IM sugiere dos periodos de formación de 


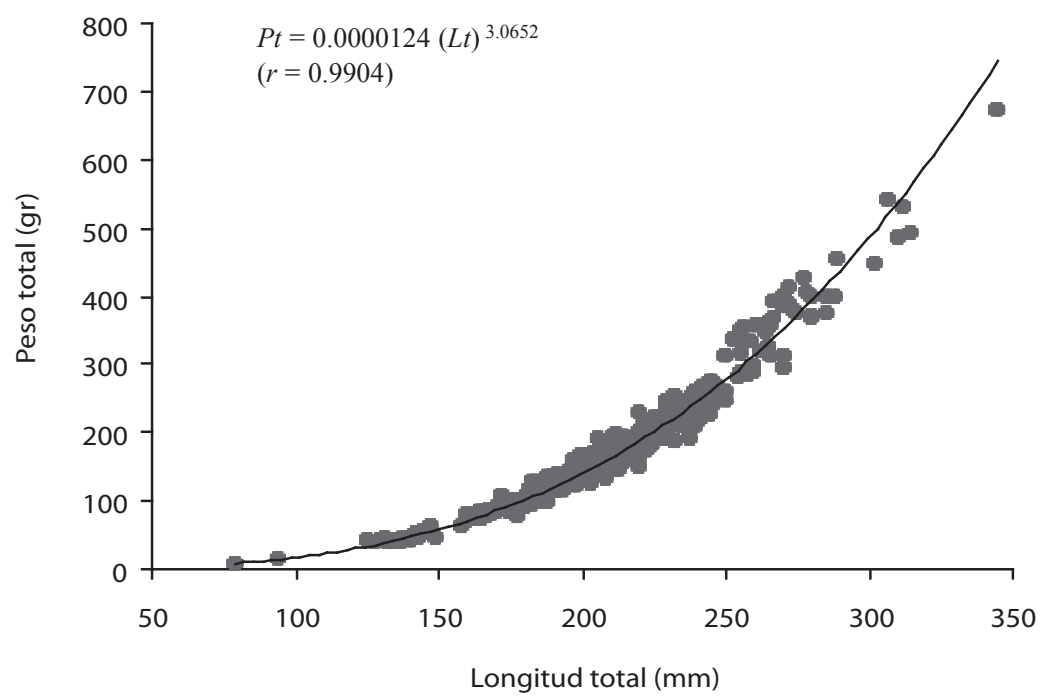

Fig. 2. Relación longitud total-peso total.

Fig. 2. Weight-length relationship.

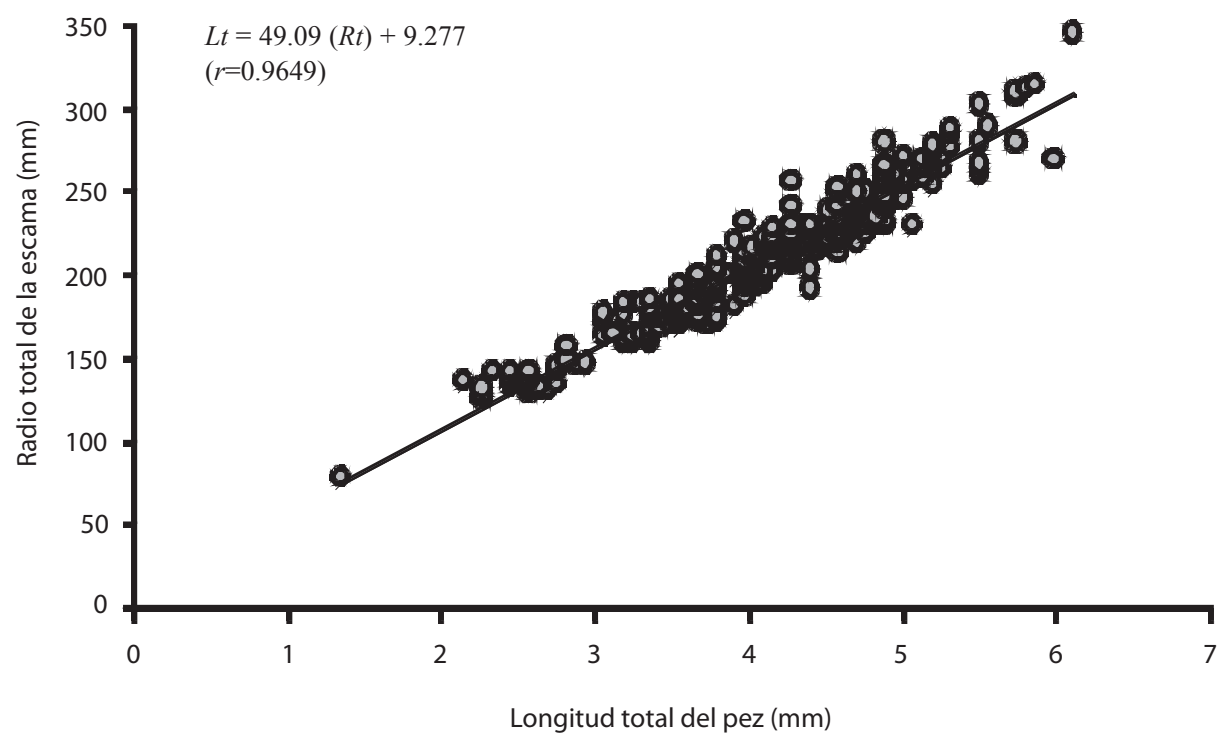

Fig. 3. Relación entre la longitud total del pez y radio total de la escama.

Fig. 3. Total fish length versus scale ratio. 


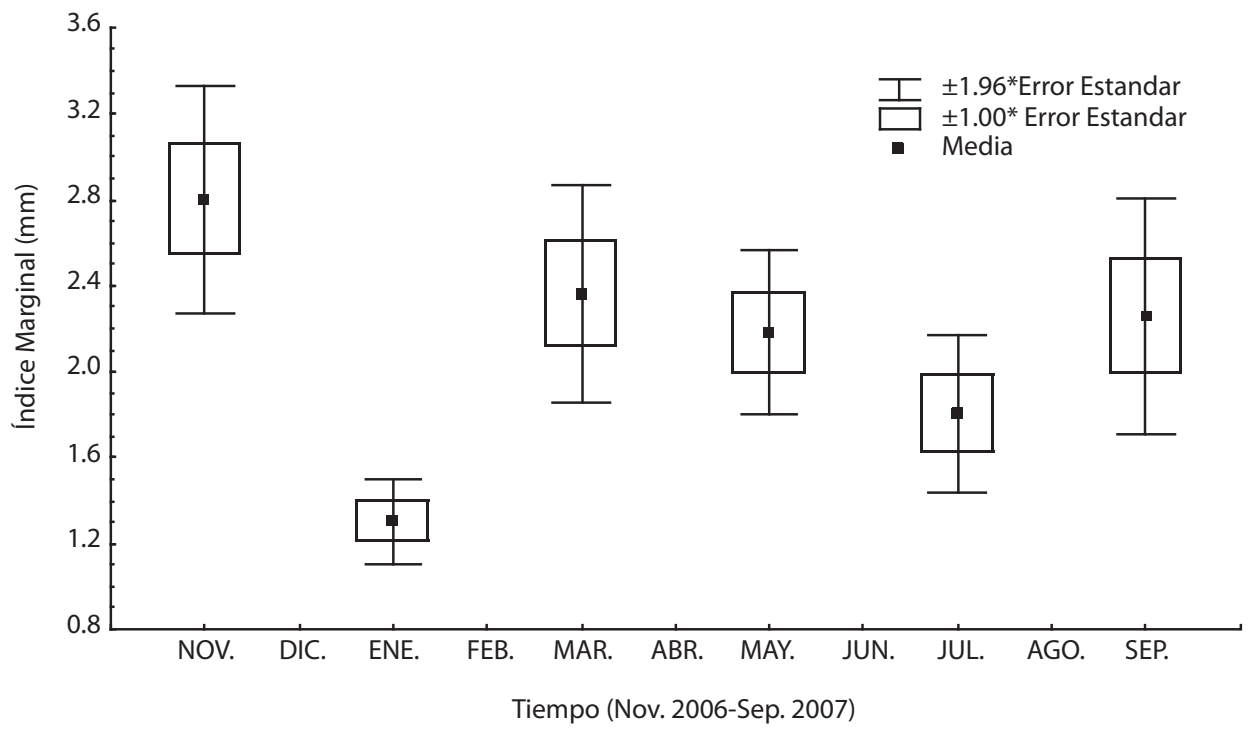

Fig. 4. Índice marginal en el tiempo.

Fig. 4. Marginal index in time.

marcas de crecimiento en las escamas de $O$. aureus durante un ciclo anual.

Índice gonadosomatico (IG): Al analizar el comportamiento del IG (Fig. 5), se observan valores bajos en los meses invernales de enero a marzo y a partir de este último mes se observó un incremento en la maduración hasta registrar los valores más altos de julio a septiembre, lo cual indica que en estos últimos meses se presentó un periodo de reproducción importante para esta especie. Los valores altos en la maduración de los organismos coinciden con el segundo periodo de formación de marca identificado a través del índice marginal, por lo que pudiera estar asociado en parte a este proceso metabólico.

Edad y crecimiento: A partir de las longitudes calculadas a cada marca de crecimiento en las escamas y de su formación semestral, se construyó la clave edad-longitud (Cuadro 1). Fueron tres años como edad máxima registrada. Una vez calculados los parámetros de crecimientos (Cuadro 2) se obtuvieron las curvas
CUADRO 1

Correspondencia de edad y longitud calculada para hembras, machos y el total de los peces evaluados

TABLE 1

Age-length relationship for males, females and total of evaluated fish

\begin{tabular}{cccc} 
Edad & \multicolumn{3}{c}{ Longitud total $(\mathrm{mm})$} \\
$\begin{array}{c}\text { (Años) } \\
0.5\end{array}$ & Hembras & Machos & Total \\
1 & 126 & 125 & 126 \\
1.5 & 177 & 179 & 179 \\
2 & 217 & 219 & 219 \\
2.5 & 247 & 249 & 248 \\
3 & 272 & 273 & 273 \\
& 304 & 306 & 305 \\
\hline
\end{tabular}

CUADRO 2

Parámetros de crecimiento calculados (von Bertalanffy)

TABLE 2

Calculated growth parameters (von Bertalanffy)

$\begin{array}{lcccc} & \mathrm{L} \infty & \mathrm{K} & \mathrm{t} 0 & \sum l^{2} \\ \text { Hembras } & 428.4 & 0.3426 & -0.5318 & 47.64 \\ \text { Machos } & 414.6 & 0.3747 & -0.4752 & 68.44 \\ \text { Total } & 416 & 0.3682 & -0.4975 & 60.48\end{array}$




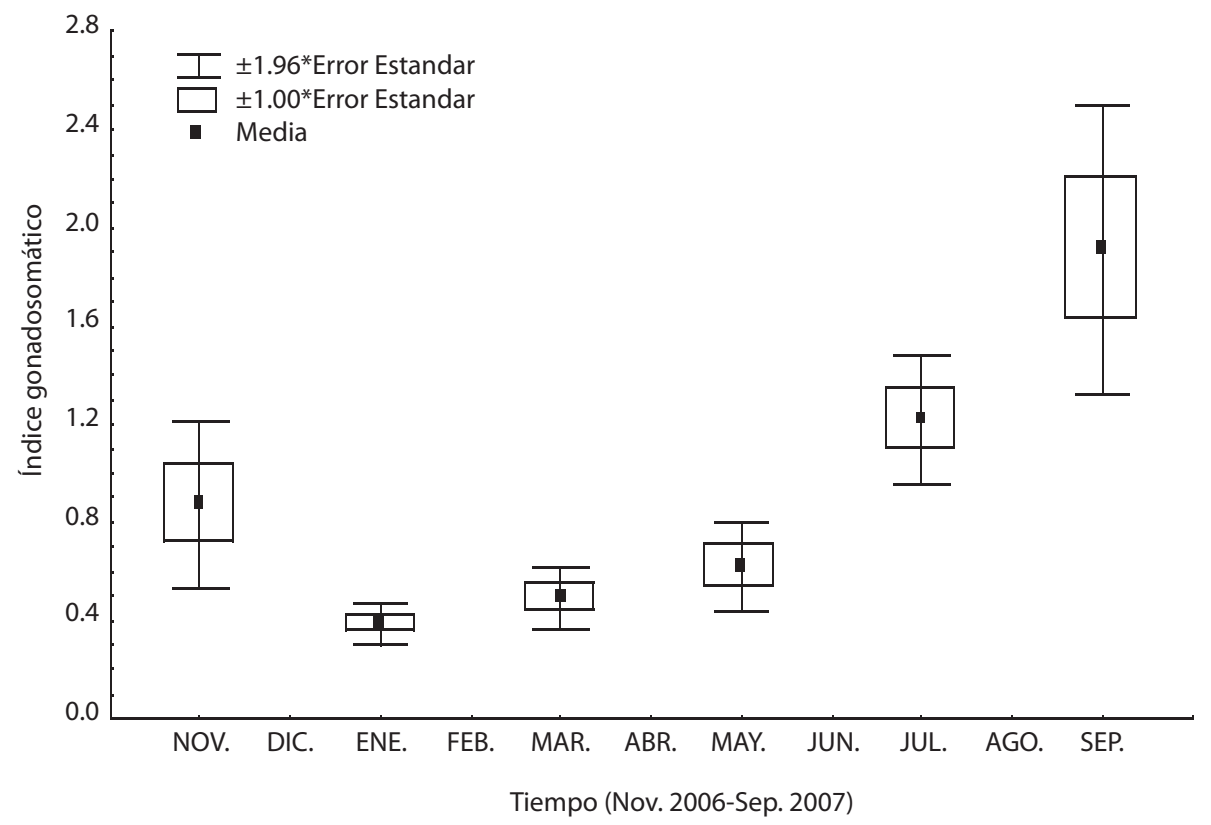

Fig. 5. Índice gonadosomático en el tiempo.

Fig. 5. Gonadosomatic index in time.

de crecimiento teóricas de longitud mediante el modelo de von Bertalanffy, dando las siguientes ecuaciones:

Para machos:

$$
L_{t}=414.6\left[1-l^{-0.3747(t+0.4752)}\right]
$$

Para hembras: (Fig. 6.)

$$
L_{t}=428.4\left[1-l^{-0.3426(t+0.5318)}\right]
$$

Modelo de crecimiento en peso para machos:

$$
P_{t}=1428\left[1-l^{-0.3426(t+0.5318)}\right]^{3.0774}
$$

Para hembras: (Fig. 7)

$$
P_{t}=1321\left[1-l^{-0.3747(t+0.4752)}\right]^{3.0807}
$$

\section{Confiabilidad de los parámetros de cre-}

cimiento: En el Cuadro 3 se observa la relación existente de los parámetros de crecimiento como longitud infinita $\left(L_{\infty}\right)$ y la tasa de crecimiento (" $K$ ") para $O$. aureus obtenidos en el presente trabajo, y los reportados en la literatura.

\section{DISCUSIÓN}

Al comparar las distribuciones de frecuencia en longitud para machos y hembras se encontró que existen diferencias significativas entre sexos, y se demostró estadísticamente que los machos son más grandes que las hembras. Lo anterior coincide con lo señalado por Sánchez (2000) en la presa Huites, Sinaloa, y con Ramírez (2007) en la presa El Varejonal, Sinaloa, México donde los machos fueron significativamente mayores que las hembras.

El análisis de la relación longitud-peso mostró un valor de la pendiente $(b=3.065)$, lo que resultó estadísticamente mayor que 3 , indicando un crecimiento de tipo alométrico positivo. Este mismo tipo de crecimiento ha sido reportado por Ramírez (2007) en la Presa Adolfo López Mateos, El Varejonal. Sin embargo, lo anterior difiere con lo reportado para esta especie por Beltrán et al. (1997) en la Presa Bacurato y Flores (2006) en la presa El Salto, ambas en el estado de Sinaloa, México, 

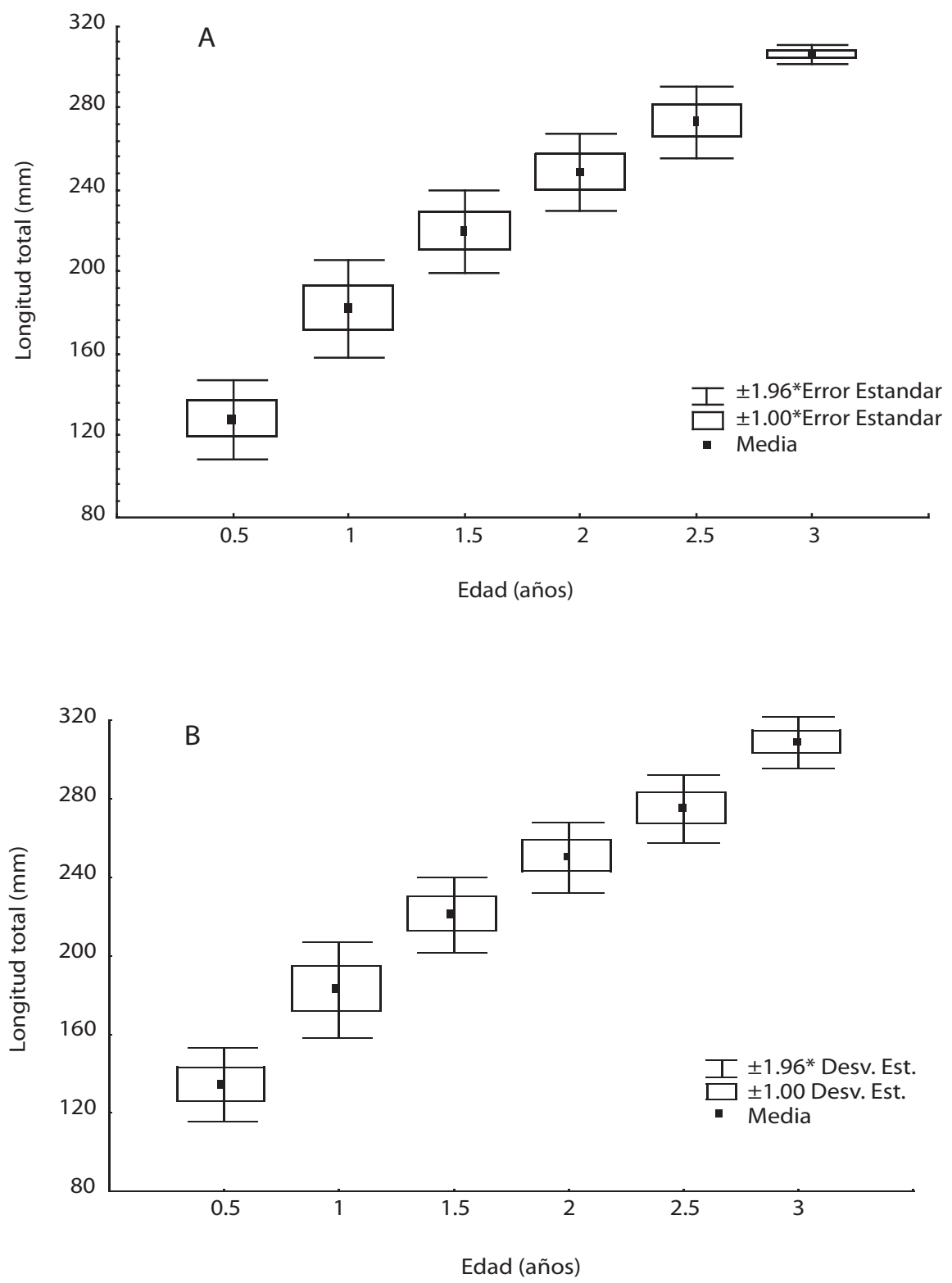

Fig. 6. Curva de crecimiento en longitud: A) machos y B) hembras.

Fig. 6. Growth curve in length: A) males and B) females. 

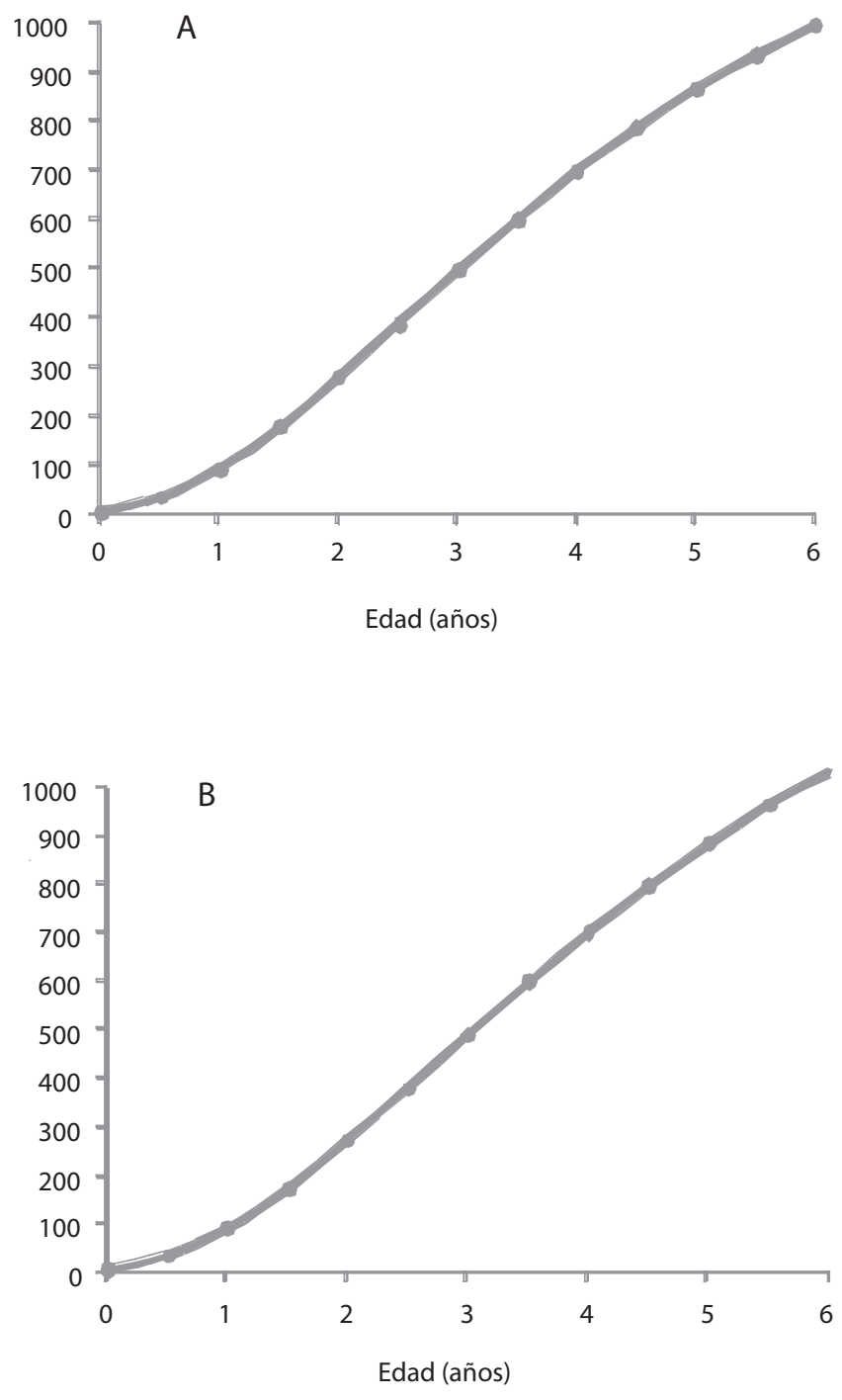

Fig. 7. Curva de crecimiento en peso para machos (A) y hembras (B).

Fig. 7. Growth curve in weight: A) males and B) females.

quienes señalan que el tipo de crecimiento presentado por estos organismos resultó ser de tipo isométrico, tal diferencia probablemente se debe en gran medida al rango de tallas analizadas. Estudios realizados en distintas especies y ambientes muestran que el valor de la pendiente de esta relación oscila entre $2.5 \mathrm{y}$ 3.5 (Granado 1996).
Retrocálculo de longitudes pretéritas: Para realizar el retrocálculo de longitudes pretéritas es necesario conocer la relación de la escama respecto a la longitud del pez, lo cual permite conocer la historia del crecimiento individual de los peces; existe una proporcionalidad entre el crecimiento somático y el crecimiento de la estructura dura del organismo 


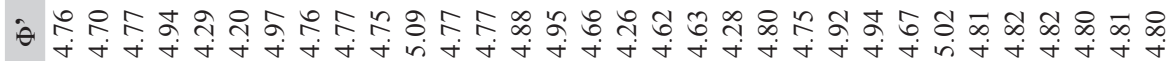

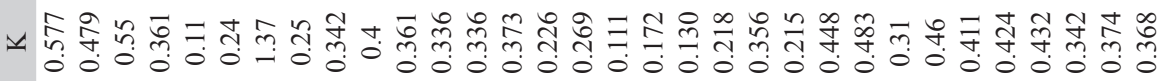

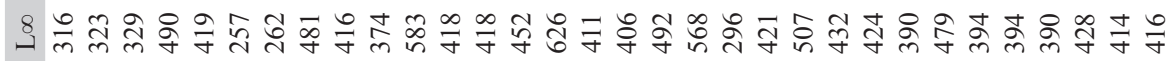

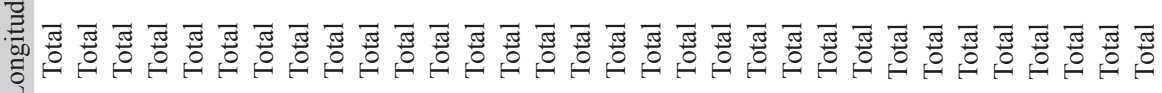

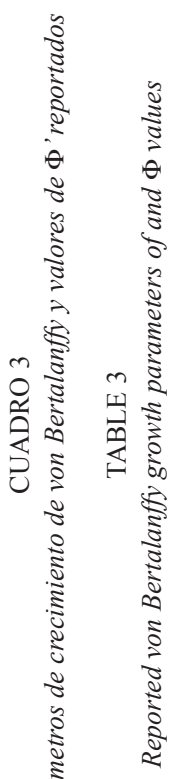

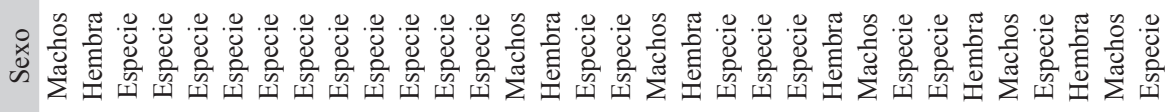

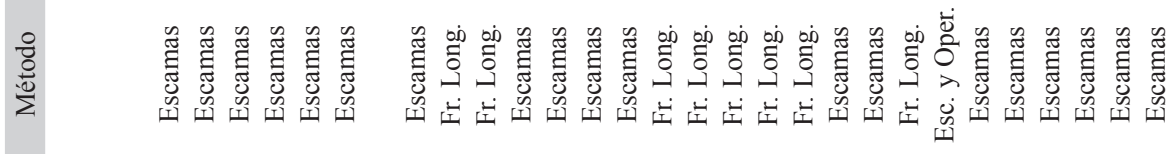

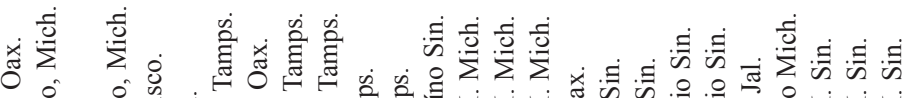

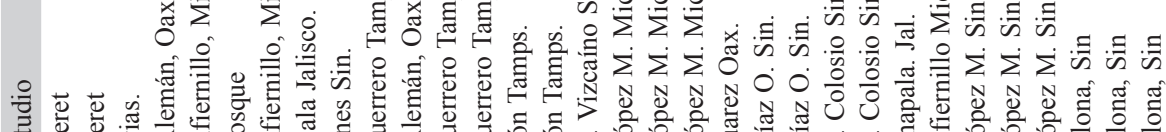

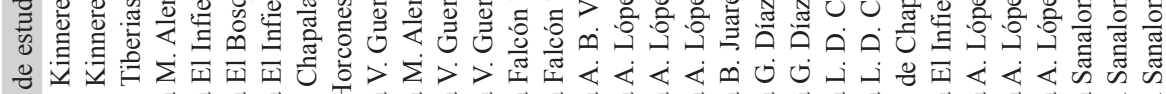

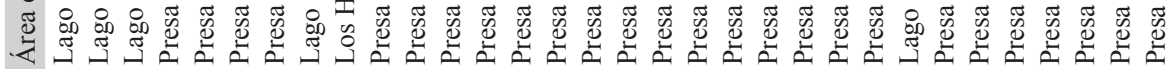

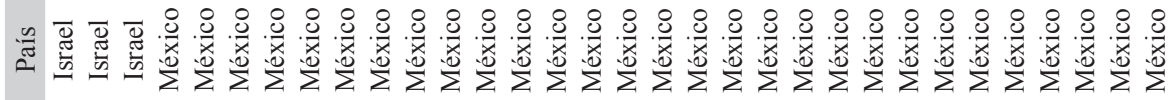

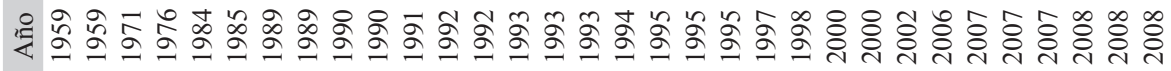

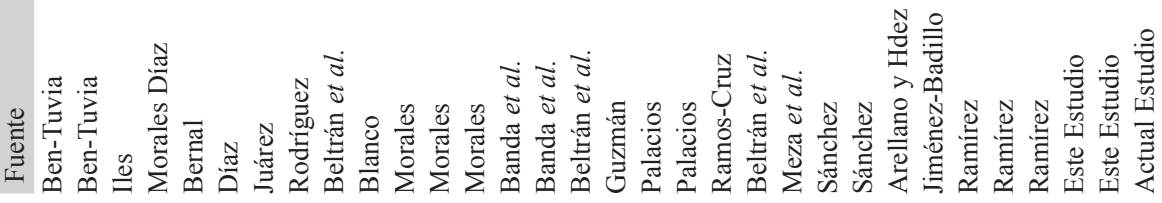


analizado (Hare \& Cowen 1995, Meekan et al. 1998, Sirios et al. 1998). En el presente trabajo la relación de la longitud del radio total de la escama $\left(R_{t}\right)$ y la longitud total del pez $\left(L_{t}\right)$, resultó ser de tipo lineal altamente significativa entre las variables.

El análisis retrospectivo o retrocálculo es una técnica que permite estimar dimensiones corporales en edades o etapas previas de la historia de vida de los peces. Existen dos supuestos subyacentes para su aplicación: 1) periodicidad constante de los incrementos y 2) proporción constante entre el crecimiento de la estructura dura y el crecimiento somático del pez (Hare \& Cowen 1995, Meekan et al. 1998, Sirois et al. 1998). En el presente trabajo se cumplió con los supuestos mencionados y de esta manera se logró estimar la longitud total promedio correspondiente a cada grupo de edad al aplicar esta técnica. De esta manera se encontraron 6 grupos de edad observándose cierta variación en las longitudes calculadas a cada grupo de edad la cual fue mayor en los primeros grupos, disminuyendo paulatinamente en los grupos subsecuentes. Una posible causa de esta variación, además de la variabilidad natural de los peces, es que en los cálculos de los grupos de edad se incluyeron organismos que nacieron en tiempos diferentes ya que $O$. aureus extiende su periodo reproductivo en varios meses del año, por otro lado las repoblaciones del embalse con crías de tilapia debieron haber influido en este comportamiento. Ibáñez \& Romo-Coronel (2007), señalan que las marcas de crecimiento a anuli en escamas de la tilapia muestran mucha variabilidad en los organismos que son criados en granjas lo que se puede deber al tipo de alimento que reciben o a que son manipulados en los estanques de la granja.

\section{Validación de las marcas de crecimien-}

to: En estudios sobre edad que involucran la lectura de cualquier estructura dura de los peces como las escamas es de suma importancia la validación de la aparición de las marcas de crecimiento, es decir, la asignación de una temporalidad a la formación de los anillos
(Carlander 1982, Casselman 1983, Beamish \& McFarlane 1987). Para validar la aparición de las marcas de crecimiento en las escamas de $O$. aureus en el presente estudio, se realizó el seguimiento del índice marginal promedio mensual (I.M.) y se corroboró mediante el índice gonadosomático. El índice marginal mostró dos periodos de formación de las marcas de crecimiento, el primero durante el invierno entre los meses de diciembre a febrero, con una reducción significativa de su ritmo metabólico. Un segundo periodo de formación de marcas (junio-agosto), pudiera estar relacionado con altas temperaturas del agua y la estratificación térmica intensificada, que restringe la solubilidad del oxígeno disuelto en el agua en los primeros seis metros; otro factor es la gran competencia por espacio y alimento originada por la disminución del volumen de agua en el embalse debido al estiaje, y en el que el área de 2900 pasa a solo 900 ha (un 69\% ) (Beltrán et al. 2007). Un factor más que probablemente se suma a los anteriores, sobre todo en organismos sexualmente maduros, es la reproducción, que a juzgar por el índice gonadosomático presenta los valores más altos de julio a septiembre. Este último factor contribuye de manera significativa en la formación de marcas en las escamas debido a que además de la desviación de energía en la maduración de las gónadas, las tilapias limitan la alimentación durante la incubación bucal y crianza de los alevines con la consecuente reducción de la tasa de crecimiento. Un resultado similar ha sido reportado por otros autores en el mundo para miembros de la familia Cichlidae entre los que se pueden mencionar a Yosef \& Casselman (1995), y Admassu \& Casselman (2000) que para el lago Awassa, Etiopia, reportaron la formación de dos marcas de crecimiento por año con base al índice marginal en otolitos de O. niloticus. En México, dichas formaciones de marcas fueron asociadas a eventos reproductivos y a disminución del nivel del agua del embalse durante el estiaje: Garduño et al. (1996) en la Laguna de Coatetelco, y Morelos \& Tovar (2005) en la Presa Emiliano Zapata, Morelos, encuentran dos periodos de formación de marcas en las 
escamas, y en escamas y otolitos, respectivamente. Gómez-Márquez et al (2008), estudian la edad y crecimiento de $O$. niloticus a partir del hueso opercular, en la laguna de Coatetelco, Morelos, México, y concluyen que la formación de marcas se debe a la alta temperatura que registra el agua del embalse, a la época de estiaje, y al periodo reproductivo.

Edad y crecimiento: El crecimiento es considerado como el proceso de incremento gradual o desarrollo progresivo con el tiempo en tamaño o en peso del organismo (Tovar 2005). En el presente estudio se analizaron los parámetro de crecimiento a partir del modelo de von Bertalanffy, a través del método de mínimos cuadrados no lineales ya que este fue el que presentó la menor suma de las diferencias al cuadrado $\left(\sum \mathrm{e}^{2}\right)$. Mediante este método se estimó una longitud máxima teórica $\left(L_{\infty}\right)$ ligeramente mayor en hembras $(428 \mathrm{~mm})$ que en machos $(415 \mathrm{~mm})$, mientras que la tasa de crecimiento $(K)$ fue menor en hembras (0.3426) que en machos (0.3747). Aunque la diferencia en estos parámetros no es significativa, el valor relativamente mayor del coeficiente " $\mathrm{K}$ " en los machos indica que estos pueden alcanzar en un menor tiempo una talla cercana a la longitud máxima teórica. Una tasa metabólica mayor en machos de $O$. aureus, ha sido reportada por varios autores entre los que se puede mencionar a Ben-Tuvia (1959); Banda \& Blanco (1993); Palacios (1995); Sánchez (2000); Ramírez (2007).

Confiabilidad de los parámetros de crecimiento: Los parámetros de crecimiento encontrados en este estudio indican valores de índice ligeramente menores a los obtenidos Bernal (1984), Díaz (1985) y Guzmán (1994) quienes a través de la lectura de escamas de la mojarra $O$. aureus en la presa A. López Mateos, Michoacán, estimaron menores tasas de crecimiento con menores valores de $\Phi$ '. Por otra parte, valores mayores han sido reportados por Morales (1992) en la Presa Miguel Alemán, Oaxaca, Juárez (1989) y Jiménez (2006) en la Presa Adolfo López Mateos Infiernillo,
Michoacán. Con respecto a las Presas de Sinaloa, los valores de $\Phi$ ' de este estudio son muy similares a lo reportado por Beltrán et al. (1997) en la presa Gustavo Díaz Ordaz Bacurato, y por Ramírez (2007) en la presa Adolfo López Mateos El Varejonal, pero resultaron menores a lo que señala Sánchez (2000) para la presa Luís Donaldo Colosio Huites, tal diferencia probablemente se debe al número de grupos de edad analizados en este embalse, los cuales son el resultado de la presión ejercida por la pesca comercial.

\section{AGRADECIMIENTOS.}

Al Gobierno del Estado de Sinaloa, a las Sociedad Cooperativa de Producción Pesquera "Sanalona" por el apoyo recibido durante la realización del presente trabajo y a Arturo Núñez Pasten por la revisión del manuscrito

\section{RESUMEN}

Oreochromis aureus es un importante recurso pesquero en el embalse, por lo cual se realizó un estudio sobre su edad y crecimiento desde noviembre del 2006 a septiembre del 2007. Se realizaron muestreos bimensuales con redes agalleras de varios tamaños de luz de malla, y también se colectaron peces de la captura comercial. Se midió la longitud total $\left(\mathrm{L}_{\mathrm{t}}\right)$, el peso total $\left(\mathrm{P}_{\mathrm{t}}\right)$, el sexo y la madurez gonádica. La edad se determinó mediante lecturas de marcas de crecimiento en escamas y su validación se realizó mediante el índice marginal e índice gonadosomático. Se colectaron 384 peces, las tallas y pesos variaron de 125 a $345 \mathrm{~mm}$ y de 40 a $671 \mathrm{~g}$, respectivamente. El crecimiento en longitud y peso se describe mediante las ecuaciones de von Bertalanffy: $\mathrm{L}_{\mathrm{t}}=416\left[1-\mathrm{e}^{-0.3682(\mathrm{t}+0.4975)}\right]$ y $\mathrm{P}_{\mathrm{t}}=1323[1-$ $\left.\mathrm{e}^{-0.3682(t+0.4975)]}\right]^{3.0652}$. Se determinaron dos marcas de crecimiento durante el año. Estas fueron asociadas con factores metabólicos y medioambientales como la reproducción y la oscilación de temperatura, combinada con procesos de mezcla y disminución de los niveles del agua del embalse.

Palabras claves: edad, crecimiento, Oreochromis aureus, embalse, México.

\section{REFERENCIAS}

Admassu, D. \& J.M. Casselman. 2000. Otolith age determination for adult tilapia Oreochromis niloticus L. from Lake Awassa (Etiopian Rift Valley) by interpre- 
ting annuli and differentiating biannual recruitment. Hidrobiologia 418: 15-24.

Bagenal, T.B. \& F.W. Tesch. 1978. Age and growth, p. 101-136. In T. B. Bagenal (ed.). Methods for assessment of fish production in fresh waters, Blackwell, Oxford.

Beamish, J.R. \& G.A. McFarlane. 1987. Current trends in age determination methodology, p. 15-42. In Summerfelt R.C. \& G. Hall (eds.). The age and growth of fish. Iowa State University Press, Iowa, EEUU.

Beltrán, A.R., P.J. Sánchez, L.J.P Ramírez, B.G. Arroyo \& E.H. Galaviz. 1997. Informe final del proyecto: estudio para evaluar las condiciones hidrobiológicas y el estado actual de la pesquería en la presa Gustavo Díaz Ordaz "Bacurato", UAS-SEP. Sinaloa, México.

Beltrán, A.R. 2003. Las aguas continentales de Sinaloa, p. 159-183. In J. Karma \& J. L. Baraud, Sinaloa y su ambiente (ed.). Sinaloa y su ambiente: visiones del presente y perspectivas. UAS. Sinaloa, México.

Bernal, B.F. 1984. Análisis de los factores relacionados con la producción pesquera de Tilapia nilotica en la Presa Adolfo López Mateos (Infiernillo), Michoacán-Gro. Tesis de Licenciatura. UNAM, México.

Carlander, K.D. 1974. Difficulties in ageing fish in relation to inland fishery management, p. 200-205. In T.B. Bagenal (ed.). The ageing of fish. Unwin Brothers, Surrey, Inglaterra.

Carlander, K.D. 1982. Standard intercepts for calculating lengths from scale measurements for some centrarchid and percid fishes. Trans. Amer. Fish. Soc. 111: 332-336.

Casselman, J.M. 1983. Age and growth assessment of fishes from their calcified structures-techniques and tools. U.S. Dep. Commer., NOAA Tech.Rep. NMFS8: 1-17.

Contreras-MacBeath, T. Mejia- Mojica \& R. Carrillo-Wilson. 1998. Negative impact on the aquatic ecosystems of the state of Morelos Mexico from introduced aquarium and other commercal fish. Aquarium Sciences and Conservation 2: 67-78.

Díaz, L.M. 1985. Contribución al conocimiento de edad y crecimiento de Sarotherodon aureus (mojarra) de la presa "El Bosque", la Encarnación, Michoacán. Tesis de Licenciatura. UNAM, México.

Flores, C. 2006. Análisis de los efectos de la selectividad de las redes agalleras sobre algunos aspectos de la pesquería comercial (Oreochromis aureus), en la presa Aurelio Benassini Vizcaíno "El Salto", Sinaloa,
México. Tesis de Licenciatura, Facultad de Ciencias del Mar, Universidad Autónoma de Sinaloa. Mazatlán, Sinaloa, México.

Fraser, C. Mc L. 1916. Growth of the spring salmon. Trans. Pacif. Fish. Soc. Seattle 1915: 29-39.

Garduño, P.M. \& E.J. Avelar. 1996. Edad y crecimiento de la mojarra (Oreochromis niloticus) en la laguna de Coatetelco, Morelos. Tesis profesional. Facultad de estudios superiores Zaragoza. Universidad Nacional Autónoma de México. México D.F., México.

Gómez-Márquez, J.L., B. Peña-Mendoza, H.I. SalgadoUgarte \& J.L. Arredondo-Figueroa. 2008. Age and growth of tilapia, Oreochromis nilotus (Perciformes: Cichlidae) from a tropical shallow lake in México. Rev. Biol. Trop. 56: 875-884.

Granado, L.C. 1996. Ecología de peces. Secretariado de Publicaciones Universidad de Sevilla. Sevilla, España.

Guzmán, U.A. 1994. Parámetros biológicos de Tilapia (Oreochromis aureus Steindachner, 1864) de la presa Adolfo López Mateos "El Infiernillo" MichoacánGuerrero, México. Tesis Profesional. México D.F., México.

Jearld, A. Jr. 1983. Age determination, p. 301-324. In L.A. Nielsen \& D.L. Johnson (eds.) Fisheries techniques. Am. Fish. Soc. Bethesda, MD. Facultad de Ciencias. UNAM. México D.F., México.

Jiménez, B.L. 2006. Age-growth models for tilapia Oreochromis aureus (Perciformes, Cichlidae) of the Infiernillo reservoir, Mexico and reproductive behavior. Rev. Biol. Trop. 54: 577-588.

Hare, J.A. \& R.K. Cowen. 1995. Effect of age, growth rate, and ontogeny on the otolith sizefish size relationship in bluefish, Pomatomus saltatrix, and the implications for backcalculation of size in fish early life history stages. Can. J. Fish. Aquat. Sci. 52: 1909-1922.

Ibañez, A.L. \& M.A. Romo-Coronel. 2007. Formation of ring marks in stocked tilapia juveniles (Oreochromis aureus/O. niloticus) (Perciformes: Cichlidae). Rev. Biol. Trop. 55: 1005-1013.

Jones, C.M. 2002. Age and growth, p.33-63. In Fuiman Lee A. \& R.G. Werner (eds). Fishery Science, The unique contributions of early life stages. Blackwell Science, Oxford, Reino Unido.

Lee, R.M. 1920. A review of the methods of age and growth determinations by means of scales. Fishery Invest. Lond. Ser. II 4 (2): 32. 
Meekan, M.G., J.J. Dodson, S.P. Good \& D.A.J. Ryan. 1998. Otolith and fish size relationships, measurement error, and size-selective mortality during the early life of Atlantic salmon (Salmo salar). Can. J. Fish. Aquat. Sci. 55: 1663-1673.

Morales, D.A. 1991. La tilapia en México biología, cultivo y pesquerías. AGT, S.A. México, D.F. México.

Morales-Nin, B. 1989. Growth determination of tropical marine fishes by means of otolith interpretation and length frequency analysis. Aquat. Living Resour. 2: 241-253.

Morales-Nin, B. 2000. Review of the growth regulation process of otolith daily increment formation. Fish. Research 46: 53-67.

Pannella, G. 1974. Otolith growth patterns: an aid in age determination in temperate and tropical fishes, $\mathrm{p}$. 28-39. In T.B. Bagenal (ed.). The ageing of fish. Unwin Brothers, Surrey, Inglaterra.

Pauly, D. 1983. Algunos métodos simples para la evaluación de recursos pesqueros tropicales. FAO. Fisheries Technical Paper, 234. FAO, Roma, Italia.

Pauly, D. \& J.L. Munro. 1984. Once more on the comparison of growth in fish and invertebrates. Fishbyte 2: 121.

Ramírez, T.J.H. 2007. Edad y crecimiento de la mojarra Oreochromis aureus (Steindachner, 1864), de la presa Adolfo López Mateos "El Varejonal" Badiraguato Sinaloa, México. Tesis Profesional. Facultad de Ciencias del Mar. Universidad Autónoma de Sinaloa. Mazatlán, Sinaloa, México.

Rossenblum, M., J. Pudney \& I. Callard. 1987. Gonadal morphology enzyme histochemistry and plasma steroid levels during the annual reproductive cycle of male and female brown bullhead catfish, Ictalurus nebulosus Lesueur. J. Fish Biol. 31: 325-341.
Ruiz-Durán. M.F., A. Origel \& G. Rodríguez. 1970. Líneas de crecimiento en escamas de algunos peces de México. Inst. Nal. de Invest. Biol. Pesq. Serie Investig. Pesq., Estudio 2. México D.F., México.

Sánchez, P.J. 2000. Determinación de la edad y crecimiento de la mojarra Oreochromis aureus (Steindachner, 1864) (Pisces: Cichlidae) en la presa Luis Donaldo Colosio, Choix, Sinaloa, México. Tesis de Maestría, Facultad de Ciencias de Mar, UAS. Mazatlán, Sinaloa, México.

Sirois, P., F. Lecomte \& J.J. Dodson. 1998. An otolith-based back-calculation method to account for time-varying growth rate in rainbow smelt (Osmerus mordax) larvae. Can. J. Fish. Aquat. Sci. 55: 2662-2671.

Tesch, F. W. 1968. Age and Growth, p. 93-123. In W.E. Ricker (ed.). Methods for assessment of fish production in fresh waters. IBP HANDBOOK No.3. Blackwell, Oxford, Reino Unido.

Tovar, G.A. 2005. Edad y crecimiento de O. niloticus por medio de estructuras duras, Tesis profesional, Facultad de Estudios Superiores de Zaragoza, Universidad Nacional Autónoma de México. Zaragoza, México.

von Bertalanffy, L. 1938. A quantitative theory of organic growth (Inquires on growth laws II). Human Biology 10: 181-213.

Weatherley, A.H. \& H.S. Gill. 1987. The biology of fish growth. Academic. Londres, Inglaterra.

Yosef, T.G. \& J.M. Casselman. 1995. A procedure for increasing the precision of otolith age determination of tropical fish by differentiating biannual recruitment, p. 247-269. In D.H. Secor, J.M. Dean \& S.E. Campana (eds.). Recent developments in fish otolith research. University of South Carolina, Columbia, EEUU. 